J. Lake Sci. (湖泊科学), 2016, 28(3): 592-598

DOI 10. 18307/2016. 0315

(C) 2016 by Journal of Lake Sciences

\title{
夏季日最低温度升高对芬兰皮海湖浮游植物群落的影响"
}

\author{
费 磊 ${ }^{1,2}$, 邓建明 ${ }^{2}$, 秦伯强 ${ }^{2 * *}$, 朱广伟 ${ }^{2}$, 左胜鹏 ${ }^{1}$, Jouko SARVALA $^{3}$, Anne-Mari VENTEL $\ddot{A}^{4}$,
} Teija KIRKKALA ${ }^{4}$

( 1 : 安徽师范大学环境科学与工程学院,芜湖 241000)

(2: 中国科学院南京地理与湖泊研究所湖泊与环境国家重点实验室,南京 210008)

(3:Department of Biology, University of Turku, FI-20014, Turku)

(4:Pyhäjärvi Institute, Sepäntie 7, Ruukinpuisto, FI-27500 Kauttua)

摘 要: 为讨论日最低气温变化对浮游植物群落结构的影响, 以位于芬兰的皮海湖为研究对象, 选取 $1987-2013$ 年夏季 的监测数据, 采用非度量多维尺度法分析浮游植物群落结构与气象要素和理化因子之间的关系. 结果表明: 皮海湖地区 夏季增温趋势明显, 尤其是日最低气温增加显著; 皮海湖夏季浮游植物群落结构主要受气象要素影响, 其中又以日最低 气温的影响最显著. 这可能是因为浮游植物的许多生理过程存在明显的昼夜节律, 日最低气温升高能加强浮游植物的夜 间呼吸作用和对营养物质的吸收等, 从而影响浮游植物的生长和增殖速率等指标. 由于不同物种对温度的适应范围不一 致,因此日最低气温升高同样可能影响浮游植物的群落结构.

关键词: 日最低气温;皮海湖;非度量多维尺度分析;浮游植物群落

\section{Phytoplankton community response to the increasing summer daily minimum temperature in Lake Pyhäjärvi, Finland}

FEI Lei ${ }^{1,2}$, DENG Jianming ${ }^{2}$, QIN Boqiang ${ }^{2 * *}$, ZHU Guangwei ${ }^{2}$, ZUO Shengpeng ${ }^{1}$, Jouko SARVALA ${ }^{3}$, Anne-Mari VENTELÄ ${ }^{4} \&$ Teija KIRKKALA ${ }^{4}$

(1: College of Environmental Science and Engineering, Anhui Normal University, Wuhu 241000, P.R.China)

(2: State Key Laboratory of Lake Science and Environment, Nanjing Institute of Geography and Limnology, Chinese Academy of Sciences, Nanjing 210008, P.R.China)

(3: Department of Biology, University of Turku, Turku FI-20014, Finland)

(4: Pyhäjärvi Institute, Sepäntie 7, Ruukinpuisto, Kauttua FI-27500, Finland)

Abstract: To discuss the impact of the daily minimum temperature on phytoplankton community structure, the long-term relations of phytoplankton community structure with both meteorological elements and physicochemical factors in Lake Pyhäjärvi have been studied by using non-metric multidimensional scaling method to analyze the detected data in summers during 1987 - 2013 . Results from the fitted data suggested that warming trend in Lake Pyhäjärvi is significant in summer, especially the trend of daily minimum temperature. In summer, phytoplankton community structure is mainly influenced by meteorological elements, among which daily minimum temperature is the most significant. This may because many physiological processes of phytoplankton have significant circadian rhythm. The increase of daily minimum temperature can enhance the nighttime respiration and absorption of nutrients, so as to influence the growth and proliferation rates. Adaption of temperature ranges for different species is inconsistent, so the increase of daily minimum temperature is also likely to influence the community structure of phytoplankton.

* 中国科学院对外合作重点项目 (GJHZ1214)、国家自然科学基金重点项目 (41230744)、国家自然科学青年基金项 目 (41501215)、中国科学院南京地理与湖泊研究所 “一三五” 战略发展规划项目 ( NIGLAS2012135003) 和高校省级 自然科学研究重点项目 (KJ2015A122) 联合资助.2015-06-10 收稿; 2015-09-29 收修改稿. 费磊 (1991 ), 男, 硕士研究生;E-mail:feil1991@163.com.

** 通信作者;E-mail :qinbq@ niglas.ac.cn. 
Keywords: Daily minimum temperature; Lake Pyhäjärvi; non-metric multidimensional scaling; phytoplankton community

联合国政府间气候变化专门委员会 (IPCC)气候变化评估报告显示全球变暖已是不争的事实 ${ }^{\left[{ }^{[1]}\right.}$,报告同 时还指出大部分地区夜间温度增温幅度大于白天温度增温幅度 ${ }^{[2]}$, 即升温存在昼夜非对称性 ( asymmetric trends). 越来越多的研究表明当前的变暖趋势对生态系统产生了诸多不利影响 ${ }^{[3]}$, 许多陆地、淡水和海洋物 种的分布范围、季节性活动、迁徙模式、丰度以及物种间的相互作用已经发生改变. 有效减少全球变暖带来 的负面影响, 一方面要积极采取措施减缓变暖趋势, 另一方面需要了解生态系统响应全球气候变化的机制, 以便采取针对性的策略.

目前气候变化的一个显著特征是平均温度升高. 温度能控制浮游植物的很多基本功能, 是湖泊中初级 生产力的重要调节因子 ${ }^{[4]}$. 它能调节酶的活性、细胞膜的流动性以及电子转移等过程, 也能影响藻类的呼吸 和营养盐吸收, 从而影响藻类生长 ${ }^{[5]}$. 即使微小的温度波动也能影响浮游植物的光合作用和呼吸作用 ${ }^{[6]}$, 进 而影响浮游植物群落结构. 1992-2012 年的历史监测数据表明, 温度变化对太湖春季浮游植物群落演替产 生了影响, 温度上升有利于水华优势属微囊藻的生长 ${ }^{[7-8]}$, 促进了蓝藻水华的暴发 ${ }^{[9]}$. 此外, 来自大尺度调 查、模拟实验、数学模型以及古湖沼学的研究结果都证明气候变化导致的温度变化能直接或者间接引起浮 游植物群落结构的改变 ${ }^{[10]}$.

浮游植物的许多生理活动也存在明显的昼夜节律. 例如固氮蓝藻 Synechococcus sp. 固氮过程主要在晚上 进行, 而细胞分裂与光合作用主要发生在白天 ${ }^{[11]}$. 其他藻类如 Microcystis sp. 、Cyanothece sp.、Prochlorococcus sp. 等的基因转录过程分别有 $25 \% \sim 80 \%$ 表现出明显的昼夜节律 ${ }^{[12]}$, 这其中既包括调节营养盐吸收的基因, 也包括调节细胞分裂和二次代谢的基因. 由于上述昼夜节律的存在,生物对昼夜不同增温的响应也将存在 差异, 但是很多研究都忽略了这一点 ${ }^{[13]}$. 气温非对称性变化对生物的影响在许多其他生态系统, 例如森林 生态 ${ }^{[14]}$ 和农业方面的报道中已经得到证实. 基于遥感数据分析表明最低气温会影响植被指数、减少水稻产 量 ${ }^{[15-16]}$ 、影响农作物物候 ${ }^{[2]}$ 等. 不仅如此, 实验证明夜间最低气温增加也会影响土壤微生物的群落组成 ${ }^{[17]}$. 那么水温昼夜非对称性变化趋势对浮游植物群落结构影响如何? 由于广泛的报道认为全球变暖促进了蓝 藻优势, 温度的非对称性变化是否直接促进了蓝藻水华的暴发? 为了回答上述两个科学问题, 本文选取位于 芬兰西南部的皮海湖为研究对象, 探讨夏季温度非对称性变化, 尤其是日最低气温升高对浮游植物群落结 构的影响.

\section{1 材料与方法}

\section{1 研究对象}

皮海湖是芬兰西南部最大的浅水湖泊 (图 1), 面积为 $155 \mathrm{~km}^{2}$, 容量 $849 \times 10^{6} \mathrm{~m}^{3}$, 平均水深约为 $5.5 \mathrm{~m}$, 最大水深 $26 \mathrm{~m}$, 水力停留时间 $3.2 \mathrm{a}$, 流域面积 $616 \mathrm{~km}^{2[18]}$. 全年季节性气温差异显著, 每年的冰封期约为 141 d. 1990s 起湖泊解冻期内蓝藻逐渐占据优势地位. 自 1995 年皮海湖保护基金会成立后,当地政府逐渐 采取了诸如建立湿地、沉降池以及在排水区建立过滤系统等一系列修复方法来改善湖泊水质,生物调控的 方法也比较普遍, 据统计鱼类养殖可以每年减少约 $25 \%$ 的外源磷输人.

\section{2 数据篮选}

对皮海湖的监测始于 1960s 的零散监测, 但是当时监测指标较少, 完整度较低. 因此本文选取皮海湖 1987-2013 年夏季 ( 6、7、8 月) 的监测数据, 因为这一时期数据完整、详实而且连续. 监测数据包括浮游植物 生物量、理化因子和气象数据. 理化数据包括透明度 $(\mathrm{SD})$ 、总氮 $(\mathrm{TN})$ 、总磷 $(\mathrm{TP}) 、$ 电导率 ( Cond), 监测频率 为半月 1 次; 气象因素主要包括风速 (WS)、最高气温 (MaxT)、最低气温 $(M i n T)$ 和平均气温 $($ MeanT), 均为 逐日测量数据. 浮游植物监测频率与理化数据一致.

数据来源于皮海湖湖心最深处的 1 个采样点, 且理化指标和浮游植物生物量的观测位点一致. 从水体 表层到底部各层次的深度都有数据的采集, 分析的数据选择表层 $(0 \sim 5 \mathrm{~m})$ 数据, 因为 $0 \sim 5 \mathrm{~m}$ 水深基本涵盖 了全湖 $80 \%$ 的水体, 个别 $0 \sim 5 \mathrm{~m}$ 数据缺失的样本, 选取邻近的深度予以补充. 部分理化因子的监测方法在多 年间有所变化,数据预处理时取样本量最多的一组值并用线性回归进行插值和补缺. 


\section{3 研究方法}

最低气温及其他环境因子对浮游植物群落结构的影响采用非度量多维尺度分析 ( Non-metric Multi-Dimensional Scaling, NMDS) 方法完成. NMDS 是一种将多维空间的研究对象(样本或变量)简化到低维空间进 行定位、分析和归类, 同时又保留对象间原始关系的数据分析方法, 被认为是在分析群落数据时最有效的方 法之一 ${ }^{[19]}$. 排序分析使用 vegan 软件包完成, NMDS 分析由函数 metaMDS 实现 ${ }^{[20]}$. 分析数据采用逐年夏季 平均值, 分析前浮游植物生物量数据使用 Hellinger 转换, 使数据更加集中. 环境因子与排序轴的关系及显著 性 $\left(R^{2}\right.$ 和 $P$ 值) 由函数 envfit 计算得出. 浮游植物优势种使用浮游植物优势度指数 $Y_{i}$ 计算 ${ }^{[21]}$.

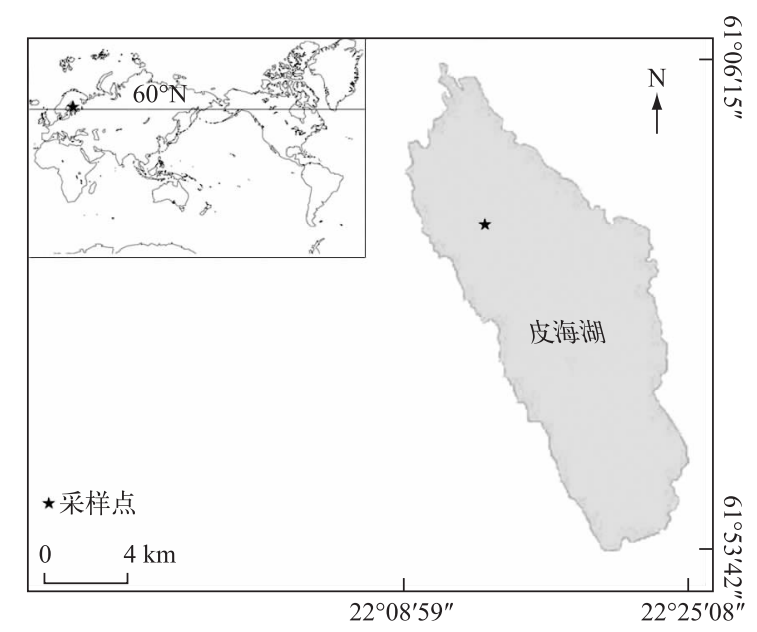

图 1 皮海湖及采样点地理位置

Fig.1 Geographic location of Lake Pyhäjärvi and sampling site

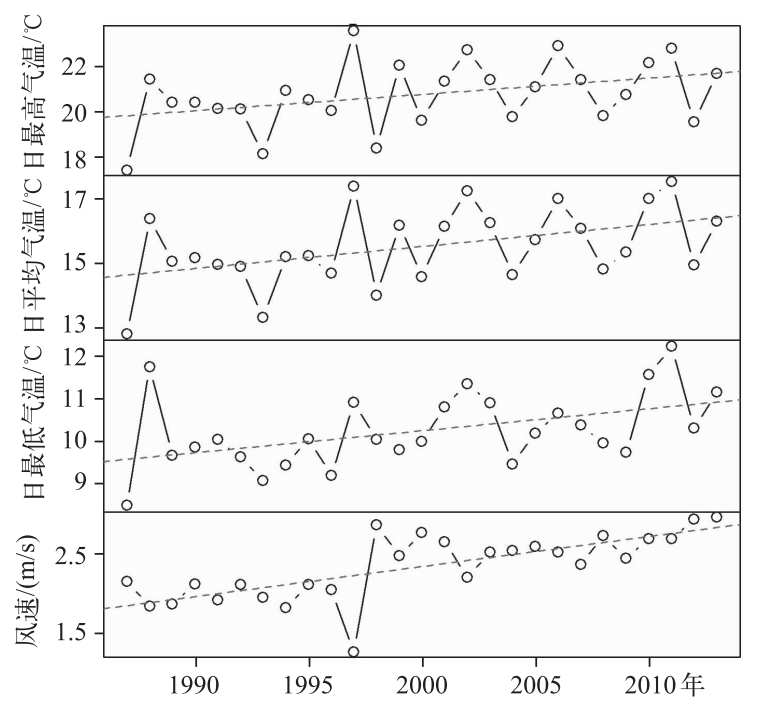

图 2 1987- 2013 年皮海湖气象因子变化趋势

Fig. 2 Annual trends of meteorological elements in Lake Pyhäjärvi during 1987 - 2013

\section{2 结果}

\section{1 气温变化}

1987- 2013 年夏季皮海湖的气温变化与全 球普遍的全球变暖现状相符合 (图 2). 日平均、 日最高和日最低气温都呈逐年上升趋势, 但各 自的增温幅度有所不同. 其中日最低温度增长 最显著 $\left(R^{2}=0.22, P<0.05\right)$, 日平均温度次之 $\left(R^{2}=0.2, P<0.05\right)$, 最高温度增温趋势最缓 $\left(R^{2}=0.15, P<0.05\right)$. 夏季风速总体呈显著升 高趋势 $\left(R^{2}=0.53, P<0.01\right)$.

\section{2 其他理化因子变化}

1987－2013 年夏季皮海湖其他理化因子都 有一定程度的变化 (图 3 ), 透明度呈逐渐下降趋 势, 下降幅度为 $0.329 \mathrm{~m} / 10$ a. 在 1990 年以前透 明度逐渐增加, 湖泊水质有逐渐变好的趋势, 在 1990 年后透明度逐渐降低, 水质状况逐渐下降.

营养盐方面, TN 和 TP 浓度波动较大, 但总 体变化并不明显. 从多年趋势来看, 电导率呈逐 渐下降趋势, 下降幅度为 $0.7 \mathrm{mS} /(\mathrm{m} \cdot 10 \mathrm{a})$. 


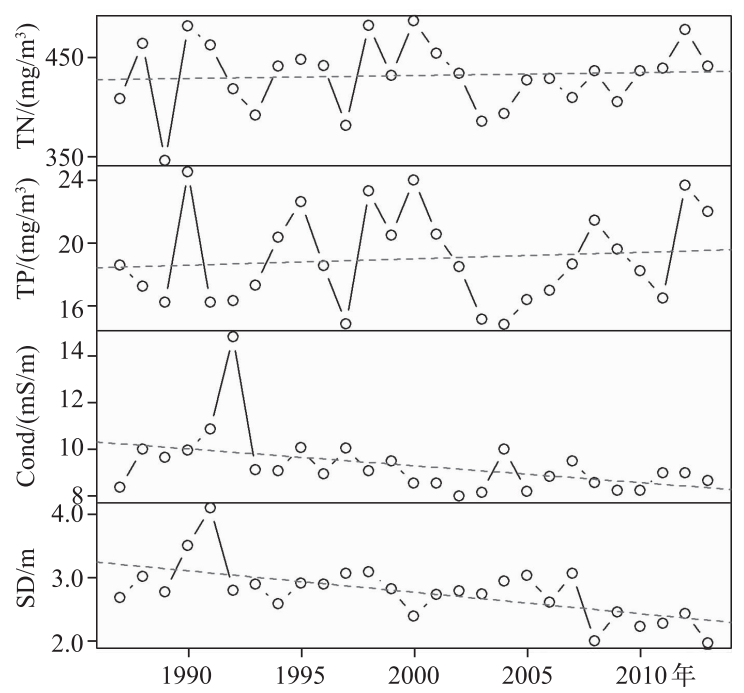

图 3 1987－2013 年皮海湖理化因子变化趋势

Fig. 3 Annual trends of physicochemical factors in Lake Pyhäjärvi during 1987 - 2013

\section{3 浮游植物群落结构变化}

1987-2013 年皮海湖浮游植物大致包括 7 门 137 属, 其中绿藻门 58 属, 隐藻门 4 属, 蓝藻门 24 属, 甲藻 门 6 属, 裸藻门 3 属, 硅藻门 41 属, 金藻门 1 属. 绿藻门、蓝藻门和硅藻门是主要的优势门类. 浮游植物夏季 生物量在 1998 年以前呈缓慢增长趋势, 1998-2000 年增长迅速并在 2001 年后迅速下降, 2007 年后呈快速 波动上升趋势. 生物量由 $1990 \mathrm{~s}$ 前的 $<1 \mathrm{mg} / \mathrm{L}$ 增长到目前的 $3 \mathrm{mg} / \mathrm{L}$ 左右 (图 4). 夏季 3 个月的浮游植物优 势种门类基本相同, 主要为蓝藻门和硅藻门, 不同月份只有少数种或属的不同, 浮游植物群落结构比较稳定.

优势度指数 $Y_{\mathrm{i}}$ 计算结果显示, 皮海湖 1987-2013 年夏季浮游植物优势种有隐藻门隐藻属 (Cryptomons) 和红胞藻属 (Rhodomonas), 蓝藻门鱼腥藻属 (Anabeana) 和浮生蓝丝藻属 (Planktothrix), 硅藻门直链藻属 (Melosira)、脆杆藻属 (Fragilaria)、双菱藻属 (Surirella) 和平板藻属 (Tabellaria) 等.

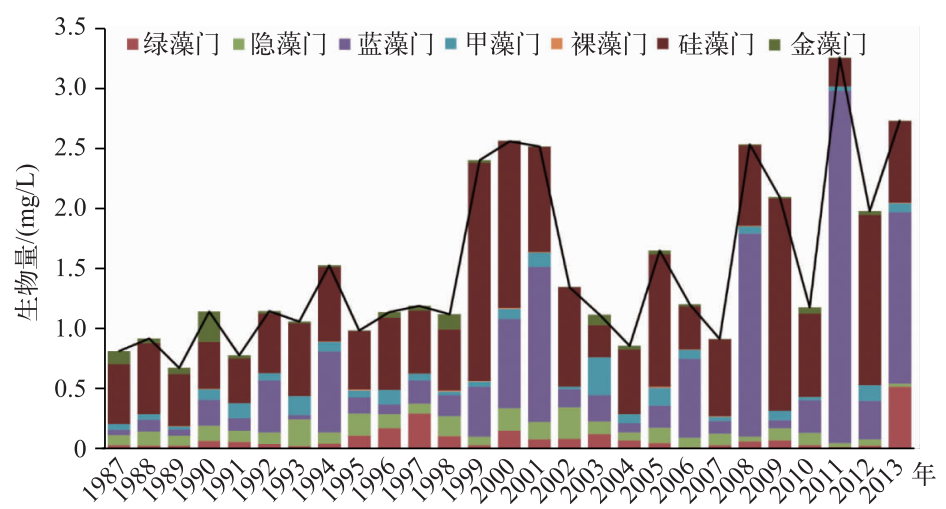

图 4 1987-2013 年夏季皮海湖浮游植物生物量及物种组成

Fig.4 Phytoplankton biomass and composition of species in Lake Pyhäjärvi in the summers of 1987 - 2013

\section{4 NMDS 分析结果}

对影响因子和浮游植物生物量之间进行 NMDS 分析, 胁强系数为 0.1 , 拟合程度较好. envfit 函数显示与 浮游植物群落组成相关性最好的影响因子是风速 $(P<0.01)$ 和最低气温 $(P<0.01)$; 其次是透明度 $(P<$ 
$0.05)$ 、日平均温度 $(P<0.05)$ 和电导率 $(P<0.05)$, 其余理化指标与浮游植物群落结构的相关性则不显著 $(P>0.05)$ (图 5).

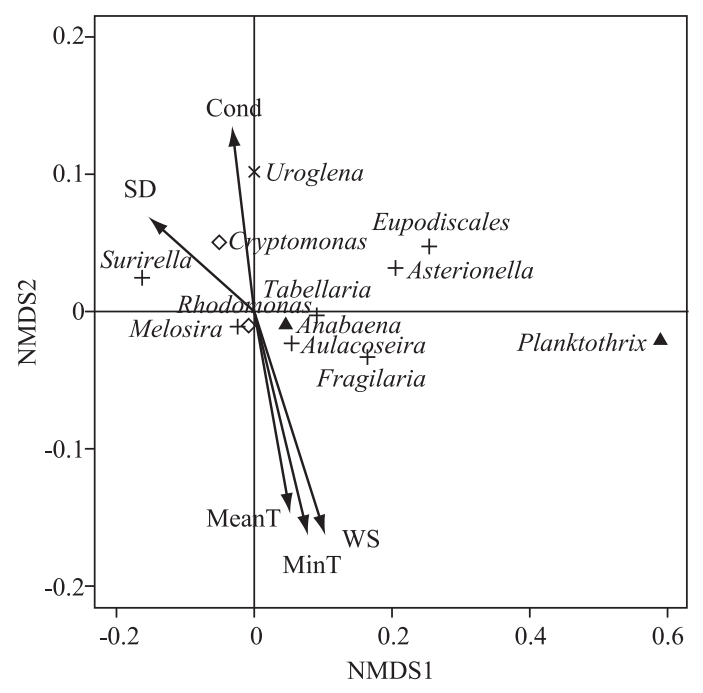

图 5 1987－2013 年皮海湖浮游植物群落结构与环境因子排序图 (只显示优势种及统计学显著的环境因子) Fig.5 Ordination diagram between phytoplankton community structure and environmental factors in Lake Pyhäjärvi during 1987-2013 ( The figure shows only the dominant species and statistically significant environmental factors)

\section{3 讨论}

\section{1 皮海湖地区的气候变化及富营养化趋势}

1987-2013 年夏季皮海湖日最低、最高和平均气温均呈逐渐上升的趋势, 但三者增温幅度不同, 这与广 泛讨论的全球气候变暖和非对称性增温相一致 ${ }^{[1]}$. 此外, 1987-2013 年皮海湖部分理化因子也有一定的变 化, 对浮游植物生长和生物量的累积都有可能产生不同程度的影响. 透明度是反映水体清澈与浑浊程度的 重要参数,能显著影响水下光照条件.

早在 1960s 皮海湖就有城市污水排人,1980s 初期磷的浓度水平逐渐升高, 在 1990 年后蓝藻在浮游植物 群落中的比例逐渐升高, 皮海湖地区湖泊富营养化逐渐加深 ${ }^{[22]}$, 这与透明度的降低时间点相符, 数据显示在 1990s 后, 透明度呈逐渐下降趋势. 进一步的分析显示, 皮海湖透明度的变化主要与风速和叶绿素 a 浓度有 关(图 6). 皮海湖属于浅水湖泊, 底泥容易受到风浪的扰动产生悬浮, 因而影响水下光照, 这与皮海湖地区 风速增加的趋势是一致的; 另外, 营养盐浓度升高或者温度增加会提高水体的初级生产力, 也会影响水下光 照. 1987- 2013 年夏季皮海湖 TP、TN 浓度变化并不显著,这与当地的湖泊管理和保护工作密切相关.

\section{2 日最低气温对皮海湖浮游植物群落结构的影响}

营养盐浓度 (尤其是磷浓度) ${ }^{[23]}$ 和水温 ${ }^{[24]}$ 等理化指标被认为是影响浮游植物群落结构的主要环境因 子 $^{[25]}$. 在皮海湖, 透明度、温度和风速是影响浮游植物群落演替的 3 个主要因素. 通过前面的分析发现, 皮 海湖地区透明度的变化主要受风速的影响. 所以, 总体来看, 1987-2013 年间夏季皮海湖地区浮游植物群落 结构的变化主要是由气象条件变化引起的. 这与在皮海湖开展的其他研究结论相一致: 该地区的模型模拟 结果显示温度变化能显著影响浮游植物 ${ }^{[26-27]}$. 分析还发现, 在所有纳人分析的温度因素中 (日最低气温、日 最高气温和日平均气温), 只有日最低气温是最显著的因素.

在以往关于气候变化对浮游植物影响的研究中, 研究者往往以平均温度为研究对象. 但是浮游植物存 在明显的昼夜节律, 温度的非对称性变化可能会对浮游植物产生不同程度的影响. 这点在陆地生态系统的 研究中得到了证实 ${ }^{[13-16]}$. 本研究结果显示, 日最低气温增加可能显著影响了浮游植物群落结构, 可能的原因 

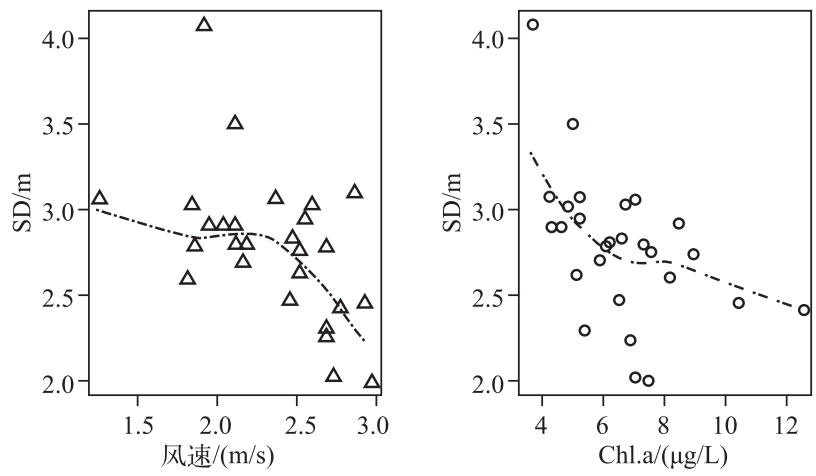

图 6 风速和叶绿素 a 浓度对皮海湖透明度的影响

Fig.6 Effect of wind speed and chlorophyll-a concentration on transparency in Lake Pyhäjärvi

是浮游植物生理活动存在明显的昼夜节律 ${ }^{[12]}$, 因此夜间温度显著增加会影响浮游植物在夜间进行的生理活 动: 首先, 最低温度升高可能会影响浮游植物的夜间呼吸作用 ${ }^{[28]}$ 和营养盐的吸收 ${ }^{[12]}$. 一方面, 呼吸作用增强 产生的能量可以供给细胞生长, 吸收的营养盐可以供给白天光合作用及细胞分裂等活动; 另一方面, 呼吸作 用的增强会消耗光合作用产生的碳水化合物, 可能造成含碳物质的减少. 但是, 同样的增温过程对不同物种 生命过程的影响可能是不一样的, 这是因为特定藻类对温度升高的响应取决于该藻类的耐受范围与温度变 化范围之间的关系 ${ }^{[29]}$. 也就是说不同物种对气候变化的响应不相同 ${ }^{[30]}$. 因此, 和平均温度影响浮游植物群 落结构一样,最低温度升高可能也会影响藻类的群落变化.

总之, 对于夜间温度增加对浮游植物, 乃至水生生态系统的影响及其机理尚不充分, 需要进一步开展 研究.

致谢: 本文浮游植物监测数据来自皮海湖研究所,气象因子数据来自芬兰气象局,理化因子数据来自芬兰环 境研究所,在此一并致谢.

\section{4 参考文献}

[ 1 ] Stocker T. Intergovernmental Panel on Climate Change. Climate change 2013: the physical science basis: Working Group I contribution to the Fifth Assessment Report of the Intergovernmental Panel on Climate Change. Climate change 2013: the physical science basis: Working Group I contribution to the Fifth Assessment Report of the Intergovernmental Panel on Climate Change, 2014.

[ 2 ] 谭凯炎, 房世波, 任三学等. 非对称性增温对农业生态系统影响研究进展. 应用气象学报, 2009, 20:634-641.

[ 3 ] Field CB, Barros VR, Mastrandrea MD et al. Ove Climate Change 2014: Impacts, Adaptation, and Vulnerability. Part A: Global and Sectoral Aspects. Contribution of Working Group II to the Fifth Assessment Report of the Intergovernmental Panel on Climate Change WG II ed. Cambridge: Cambridge University Press, 2014.

[ 4 ] Harris G. Phytoplankton ecology-structure, function and fluctuation. London: Chapman and Hall, 1986.

[ 5 ] Raven JA, Geider RJ. Temperature and algal growth. New Phytologist, 1988, 110: 441-461.

[ 6 ] Staehr PA, Sand-Jensen K. Seasonal changes in temperature and nutrient control of photosynthesis, respiration and growth of natural phytoplankton communities. Freshwater Biology, 2006, 51: 249-262.

[ 7 ] Deng J, Qin B, Paerl HW et al. Earlier and warmer springs increase cyanobacterial (Microcystis spp.) blooms in subtropical Lake Taihu, China. Freshwater Biology, 2014, 59(5) : 1076-1085.

[ 8 ] Deng J, Qin B, Paerl HW et al. Effects of Nutrients, Temperature and their interactions on spring phytoplankton community succession in Lake Taihu, China. PLoS ONE, 2014, 9(12) : e113960.

[ 9 ] Duan H, Ma R, Xu X et al. Two-Decade reconstruction of algal blooms in China's Lake Taihu. Environmental Science and Technology, 2009, 43(10): 3522-3528.

[10] 邓建明, 秦伯强. 全球变暖对淡水湖泊浮游植物影响研究进展. 湖泊科学, 2015, 27 ( 1)：1-10. DOI 


\section{0. $18307 / 2015.0101$.}

[11] Mitsui A, Kumazawa S, Takahashi A et al. Strategy by which nitrogen-fixing unicellular cyanobacteria grow photoautotrophically. Nature, 1986, 323(6090) : 720-722.

[12] Straub C, Quillardet P, Vergalli J et al. A day in the life of Microcystis aeruginosa strain PCC 7806 as revealed by a transcriptomic analysis. PLoS ONE, 2011, 6: e16208.

[13] Peng S, Piao S, Ciais P et al. Asymmetric effects of daytime and night-time warming on Northern Hemisphere vegetation. Nature, 2013, 501(7465): 88-92.

[14] Piao S, Tan J, Chen A et al. Leaf onset in the northern hemisphere triggered by daytime temperature. Nature Communications, 2015, 6: 6911 .

[15] Peng S, Huang J, Sheehy JE et al. Rice yields decline with higher night temperature from global warming. Proceedings of the National Academy of Sciences, 2004, 101(27): 9971-9975.

[16] 房世波, 谭凯炎, 任三学. 夜间增温对冬小麦生长和产量影响的实验研究. 中国农业科学, 2010, 43: 3251-3258.

[17] Kim Y, Gao C, Zheng Y et al. Different responses of arbuscular mycorrhizal fungal community to day-time and night-time warming in a semiarid steppe. Chinese Science Bulletin, 2014, 59(35) : 5080-5089.

[18] Ventelä A, Tarvainen M, Helminen H et al. Long-term management of Pyhäjärvi (Southwest Finland) : eutrophication, restoration-recovery? Lake \& Reservoir Management, 2009, 4: 428-438.

[19] Minchin PR. An evaluation of the relative robustness of techniques for ecological ordination. Plant Ecology, 1987, 69(1/ 2/3) : 89-107.

[20] Oksanen J, Blanchet F, Kindt R et al. vegan: Community Ecology Package. R package version 2. 0-6. Retrieved from http://CRAN. R-project. org/package= vegan. 2013.

[21] Mcnaughton SJ. Relationships among functional properties of Californian grassland. Nature, 1967, 216(5111) : 168-169.

[22] Ventelä AM, Kirkkala T, Lendasse A et al. Climate-related challenges in long-term management of Säkylän Pyhäjärvi ( SW Finland). Hydrobiologia, 2011, 660( 1 ) : 49-58.

[23] Tilman D, Kilham SS, Kilham P. Phytoplankton community ecology : the role of limiting nutrients. Annual Review of Ecology and Systematics, 2003, 13(1): 349-372.

[24] Eppley RW. Temperature and phytoplankton growth in the sea. Fishery Bulletin, 1972, 4(70) : 1063-1085.

[25] Reynolds CS. The ecology of phytoplankton. Cambridge: Cambridge University Press, 2006.

[26] Malve O, Laine M, Haario H et al. Bayesian modelling of algal mass occurrences-using adaptive MCMC methods with a lake water quality model. Environmental Modelling and Software, 2006, 22(7) : 966-977.

[27] Pätynen A, Elliott JA, Kiuru P et al. Modelling the impact of higher temperature on the phytoplankton of a boreal lake. Boreal Environment Research, 2014, 19(1): 66-78.

[28] Ryan MG. Effects of climate change on plant respiration. Ecological Applications, 1991, 1: 157-167.

[29] Walther G. Adapted behaviour and shifting ranges of species-a result of recent climate warming? “Fingerprints" of Climate Change, 2001: 1-15.

[30] Gerten D, Adrian R. Effects of climate warming, North Atlantic Oscillation, and El Nio-Southern Oscillation on thermal conditions and plankton dynamics in Northern Hemispheric lakes. The Scientific World Journal, 2002, 2: 586-606. 